\title{
Profile of inotuzumab ozogamicin and its potential in the treatment of acute lymphoblastic leukemia
}

This article was published in the following Dove Press journal:

Blood and Lymphatic Cancer:Targets and Therapy

17 April 2014

Number of times this article has been viewed

\section{Xavier Thomas}

Hospices Civils de Lyon, Hematology, Lyon-Sud Hospital, Pavillon Marcel Bérard, France
Correspondence: Xavier Thomas Hospices Civils de Lyon, Lyon-Sud Hospital, Hematology Department, Pavillon Marcel Bérard, Bat IG, 69495 Pierre-Bénite, France

$\mathrm{Tel}+33478862235$

Fax +33472678880

Email xavier.thomas@chu-lyon.fr
Abstract: Monoclonal antibodies are likely to make a considerable contribution in the treatment of acute lymphoblastic leukemia (ALL). High expression of CD22 antigen is found on the surface of leukemia cells in ALL. Inotuzumab ozogamicin, a CD22 monoclonal antibody conjugated to calicheamicin, which has shown efficacy in patients with lymphomas, has also shown encouraging activity in relapsed or refractory ALL with a high morphologic and molecular response rate. This article summarizes the current approaches to treating ALL with inotuzumab ozogamicin, based on available data from the literature.

Keywords: monoclonal antibody, inotuzumab ozogamicin, CMC-544, acute lymphoblastic leukemia, targeted therapy

\section{Introduction}

While the prognosis of childhood acute lymphoblastic leukemia (ALL) has improved significantly over the past decades with $80 \%-90 \%$ of children cured, the prognosis for adults with ALL remains poor. Despite modern adult ALL regimens introducing pediatric inspired or even unmodified pediatric schedules and genetic dissection of the disease, almost half of younger adults will relapse after their initial treatment, ${ }^{1,2}$ and only about $10 \%$ of relapsing patients are alive at 5 years. ${ }^{3-5}$ At the time of relapse, the only curative treatment is allogeneic stem cell transplantation (SCT). ${ }^{5}$ However, most patients will require reinduction therapy prior to SCT. Currently, no standard regimen for reinduction exists. Most salvage regimens incorporate drugs that are used in the initial treatment and complete remission (CR) rates have generally been disappointing. Novel therapeutic strategies are therefore needed.

Recently, individualized and targeted therapies have been proposed. ${ }^{6}$ The best strategy intends to deliver a cytotoxic agent to the malignant cells. The concept of monoclonal antibody (MoAb) targeted chemotherapy has been explored extensively with significant preclinical successes. ${ }^{7}$ Antibody targeted chemotherapy enables a cytotoxic agent to be delivered specifically to leukemic cells by conjugating the cytotoxic agent with a MoAb that binds to a tumor-associated antigen. This strategy theoretically minimizes exposure of normal tissues to the cytotoxic agent, and results in a significantly improved therapeutic index.

CD22 antigen, a $135 \mathrm{kDa}$ type I transmembrane sialoglycoprotein, is considered to be an ideal target for conjugate delivery because of its expression profile as well as its intracellular trafficking. It is expressed specifically on cells of B lymphoid lineage, including malignant cells of B cell origin. It is observed during B cell development at 
low levels in the cytoplasm of pro- and pre-B cells, and on the cell surface of mature cells with $\operatorname{IgM}$ and $\operatorname{IgD}$ positivity. ${ }^{8}$ The function of CD22 is not entirely clear, but it is thought to be involved in the regulation of B cell functions, such as cellular adhesion and B cell homing, and may play a role in B cell activation. ${ }^{9}$ CD22 is expressed in up to $100 \%$ of mature B cell ALL and in $93 \%$ to $96 \%$ of cases of B cell precursor ALL. ${ }^{10,11}$ It is not expressed on hematopoietic stem cells or any other cells of hematopoietic or nonhematopoietic lineages. The expression profile suggests that CD22 may be an attractive target in the treatment of B cell malignancies. Another feature supporting CD22 as an appropriate therapeutic target is that it is internalized upon ligand binding. ${ }^{12,13}$ Then CD22 delivers a costimulatory signal in normal B cells and proapoptotic signals in neoplastic B cells.

This has led to the development of a variety of antibodies to CD22 that act against different epitopes. ${ }^{14}$ Among them, CMC-544, generically known as inotuzumab ozogamicin (Pfizer Oncology, New York, NY, USA), has recently been investigated for front line therapy of relapsed or refractory adult ALL. ${ }^{15}$ Inotuzumab ozogamicin is a humanized antibody targeted intravenous chemotherapy agent composed of a CD22 targeted antibody linked to calicheamicin, a potent cytotoxic antitumor antibiotic. Calicheamicin is significantly more potent than conventional cytotoxic chemotherapeutic agents of the same class and causes cell death by inducing double strand DNA breaks. Mylotarg (gemtuzumab ozogamicin) was the first immunoconjugate of calicheamicin introduced to the clinic. Inotuzumab ozogamicin is expected to have activity in all B cell malignancies that express CD22.

\section{Molecular structure of inotuzumab ozogamicin}

Inotuzumab ozogamicin (CMC-544) is a humanized $\mathrm{MoAb}$ (type $\mathrm{IgG} 4$ ) that recognizes the CD22 antigen. It is conjugated with a calicheamicin moiety, an enediyne antibiotic and toxic natural product derived from the soil microorganism Micromonospora echinospora (Figure 1). ${ }^{16}$ Calicheamicin induces cell death in its target cells by interaction with double helical DNA in the minor groove causing site specific double stranded DNA cleavage at very low concentrations. ${ }^{17}$ In vitro, calicheamicins generate diradical species after binding with DNA. This diradical 1,4-dehydrobenzene abstracts hydrogen atoms from the sugar backbone of DNA, which results in strand scission. ${ }^{18}$ The humanized MoAb was generated by grafting the complementary determining region of the murine MoAb M5/44 into a human germ line acceptor framework. Then the variant humanized IgG4 MoAb, G5/44 (selected because of the higher internalization capacity upon stimulation), was selected and covalently linked to $\mathrm{N}$-acetyl-g-calicheamicin dimethyl hydrazide (CalichDMH) via the acid labile 4-(4'-acetylphenoxy) butanoic acid linker, yielding CMC-544 or inotuzumab ozogamicin. ${ }^{19-22}$ Inotuzumab ozogamicin binds to the minor DNA groove and causes double strand DNA breaks in a relatively sequence specific and thiol dependent manner,

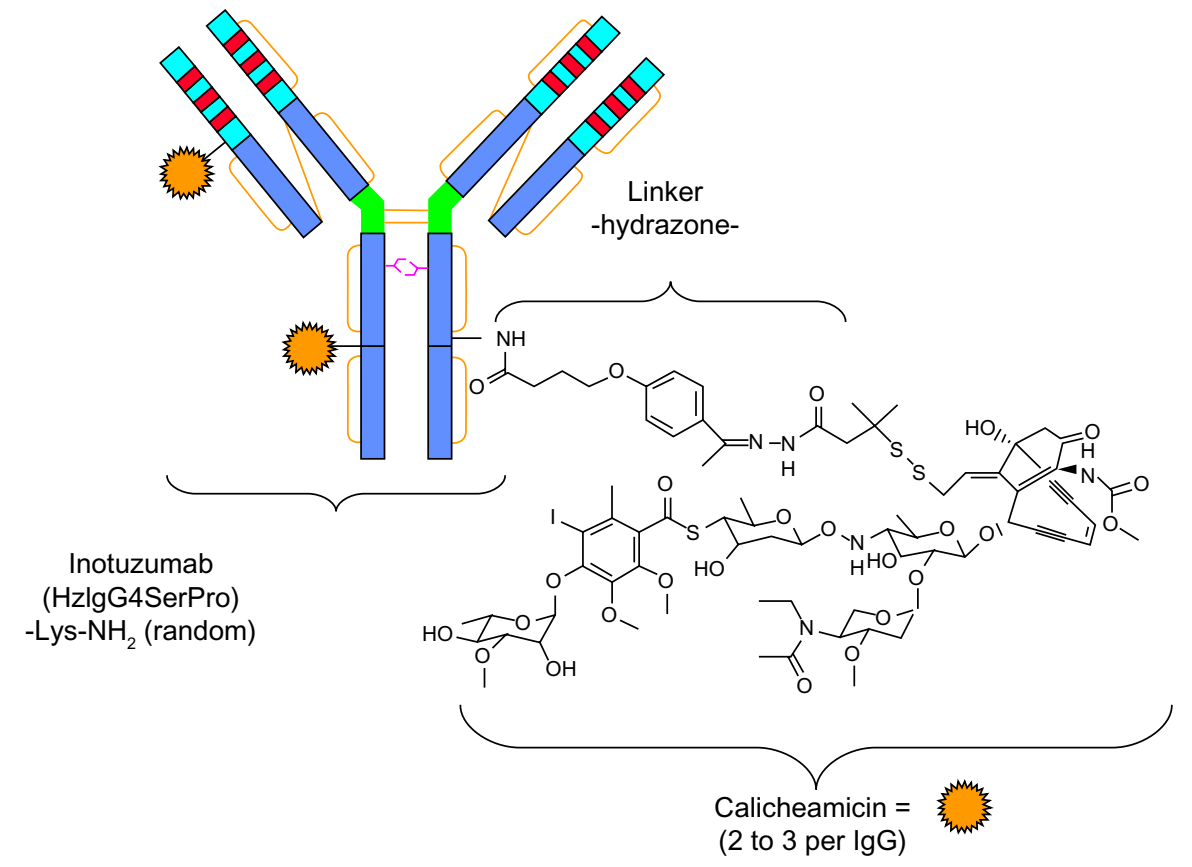

Figure I Structure of inotuzumab ozogamicin.

Notes: Inotuzumab ozogamicin is an antibody-drug conjugate that consists of a humanized monoclonal antibody inotuzumab linked to a cytotoxic agent from the class of calicheamicins. 
resulting in cell apoptosis. Inotuzumab ozogamicin is similar to gemtuzumab ozogamicin (CMC-676), except that these two conjugates recognize distinct molecular targets. Inotuzumab ozogamicin binds CD22 with subnanomolar affinity and is rapidly internalized, delivering the conjugated calicheamicin intracellularly. The internalization allows for the intracellular hydrolysis of the acetyl butyrate linker and the intracellular release of calicheamicin. ${ }^{19} \mathrm{CalichDMH}$ is a derivative of g-calicheamicin and is significantly more potent than cytotoxic chemotherapeutic agents used in cancer therapy. The conjugation to calicheamicin does not impact binding to CD22. The CD22 mediated targeted intracellular delivery of calicheamicin causes cellular arrest in the G2/M phase, followed by cell death (Figure 2).

\section{Preclinical studies with inotuzumab ozogamicin in ALL Results in vitro with cell lines and in vivo in animal models}

Inotuzumab ozogamicin has shown a potent dose dependent cytotoxicity in vitro and in vivo animal tumor models. It has been studied extensively in preclinical models of human B cell lymphoma. Both inotuzumab ozogamicin and unconjugated calicheamicin demonstrated potent cytotoxic activity against CD22 positive B cell lines. ${ }^{19}$ In vitro, inotuzumab ozogamicin has high affinity against CD22 positive ALL cell lines. ${ }^{23}$ It inhibited in vitro growth of ALL cell lines more potently than Ramos B lymphoma cells. It was also demonstrated that primary ALL cells were more sensitive to the cytotoxic effects of calicheamicin than primary acute myeloid leukemia (AML) cells or normal bone marrow cells. ${ }^{24}$ Administration to nude

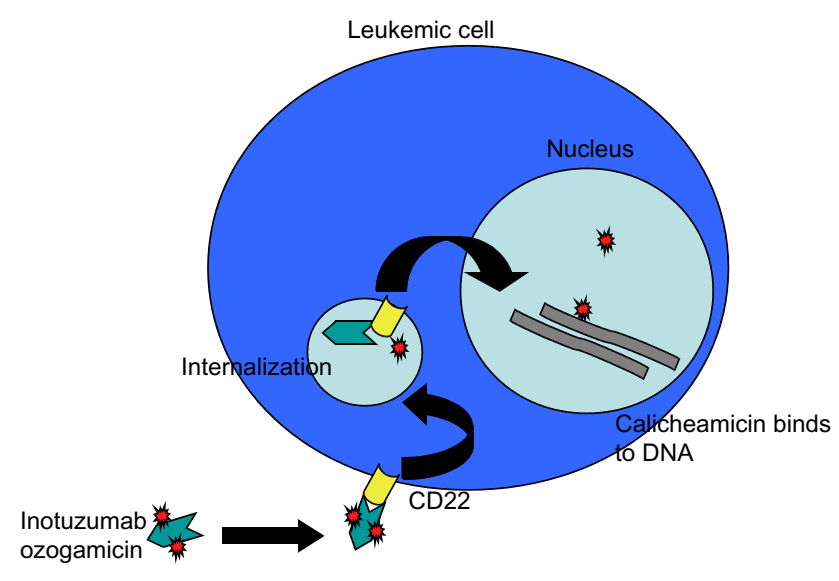

Figure 2 Mechanisms of action of inotuzumab ozogamicin.

Notes: The antibody-antigen complex is rapidly internalized (because of its subnanomolar binding affinity) upon binding to CD22. Then, calicheamicin is released inside the leukemic cell, where it binds to the minor DNA groove, inducing double stranded DNA breaks. DNA breaks are followed by apoptosis of the malignant cell. Abbreviation: DNA, deoxyribonucleic acid. mice with established subcutaneous xenografts of REH ALL cells caused dose dependent inhibition of xenograft growth producing complete tumor regression and cures in tumor bearing mice. ${ }^{25}$ When REH cells were injected intravenously with vehicle (phosphate buffered saline) and allowed to disseminate systemically, mice developed hind limb paralysis that was effectively prevented by treatment with inotuzumab ozogamicin. ${ }^{25}$ Efficacy on ALL cell lines was confirmed on pediatric B cell precursor ALL cells in vitro. ${ }^{26}$ Inotuzumab ozogamicin induced dose- and time-dependent cell death in various $\mathrm{ALL}$ cell lines, with $\mathrm{IC}_{50}$ values (defined as the concentration resulting in $50 \%$ survival) ranging from 0.15 to $4.9 \mathrm{ng} / \mathrm{mL}^{26}$

\section{Mechanisms of action and cell killing}

After binding to CD22 at the cell surface, internalization of the inotuzumab ozogamicin/CD22 receptor complex takes place. This is followed by fusion of the inotuzumab ozogamicin-containing endosome with a lysosome, resulting in a lower internal $\mathrm{pH}$ of the vesicle, subsequent degradation of the acid labile linker between G5/44 and calicheamicin, and release of calicheamicin. Severe DNA damage (frequently leading to $\mathrm{G} 2 / \mathrm{M}$ cell cycle arrest) was observed at concentrations of $100 \mathrm{ng} / \mathrm{mL}$ or higher. ${ }^{26}$ Saturation was dependent on inotuzumab ozogamicin concentration, but not on CD22 cell surface expression. Efficient cell death could already be observed at low CD22 saturation levels, indicating that, in contrast to previous results with gemtuzumab ozogamicin, no prolonged and maximal saturation is required. ${ }^{26}$ In cell lines, continuous exposure to inotuzumab ozogamicin was more effective than pulse exposure, suggesting that a continuous loop of internalization and renewed expression of CD22 contributes to the efficacy of inotuzumab ozogamicin, especially in cells with low CD22 expression. ${ }^{26}$ Inotuzumab ozogamicin also induced dose dependent cell kill in the majority of primary B cell precursor ALL cells. The efficacy was highly dependent on calicheamicin sensitivity and the CD22/inotuzumab ozogamicin internalization capacity of B cell precursor ALL, but hardly on basal and renewed CD22 expression. Although CD22 expression is required for inotuzumab ozogamicin activity, efficacy was not dependent on cell surface CD22 expression levels. Inotuzumab ozogamicin induced cell death was completely mediated via calicheamicin-induced apoptosis and not by CD22 signaling. ${ }^{23,26}$ Even low CD22 expression levels can result in high intracellular calicheamicin levels. ${ }^{26}$ This is in contrast to gemtuzumab ozogamicin, where CD33 signaling contributed to gemtuzumab ozogamicin induced cell death. ${ }^{24}$ Given the CD22 
independent uptake of inotuzumab ozogamicin, evaluation of the efficacy of this MoAb would be interesting in CD22 negative ALL patients.

\section{Mechanisms of resistance}

The heterogeneity in response to calicheamicin in ALL cells may be explained by differences in intrinsic calicheamicin sensitivity, which is dependent on the level of DNA accessibility, DNA repair capacity, or functionality of the apoptotic machinery of the target cells. In the absence of the conjugated CalichDMH, G5/44, the targeted MoAb in inotuzumab ozogamicin is ineffective in vivo as an antitumor agent in various preclinical models. ${ }^{19,27}$ The effects of inotuzumab ozogamicin may be affected by P-glycoprotein (P-gp), a membrane glycoprotein that actively pumps cytotoxic agents out of cells and decreases their intracellular concentration. Using newly established MDRI DNA transduced Daudi and Raji sublines, it was demonstrated that inotuzumab ozogamicin had no effect on the P-gp expressing sublines when compared with the parental cell lines, even though the former expressed sufficient levels of CD22. ${ }^{28}$ Multidrug resistant modifiers recovered the cytotoxic effect of inotuzumab ozogamicin in P-gp expressing sublines.

\section{Combination with rituximab}

Inotuzumab ozogamicin is regarded as an antibody targeted chemotherapy agent rather than an immunotherapeutic agent like rituximab, a chimeric human IgG1 antibody targeted to another B lymphoid lineage specific molecule, CD20. In vitro, the combination of inotuzumab ozogamicin and rituximab demonstrated an increased cytotoxic effect in $\mathrm{B}$ cell malignancies cells, supporting the rationale for the combined use of these two drugs..$^{29,30}$ Activity was enhanced up to four times with the combination, as compared with inotuzumab ozogamicin alone, suggesting synergistic activity. ${ }^{30}$ In a murine xenograft model with Ramos cells, the combination was superior in suppressing tumor proliferation and prolonging survival, as compared with the single agents. ${ }^{20}$ In vitro analyses ascribed functions such as antibody dependent cytotoxicity and complement dependent cytotoxicity to rituximab but not to inotuzumab ozogamicin, suggesting that the activity of inotuzumab ozogamicin is mediated through alternative effector functions that complement the mechanisms of rituximab induced activity. ${ }^{20}$ Increased rituximab induced cytotoxicity following prior exposure to inotuzumab ozogamicin coincided with downregulation of CD22 and CD55 molecules (both of which are closely related to resistance to rituximab), ${ }^{31}$ but preserved the level of CD20. ${ }^{29}$ In addition, inotuzumab ozogamicin was effective against human $\mathrm{B}$ cell lymphomas in murine models in which rituximab had failed as a therapeutic. ${ }^{30}$ However, in the initial clinical trials, the addition of rituximab in nine patients with CD20 positive ALL and no response after two cycles of inotuzumab ozogamicin did not offer any significant additional benefit. ${ }^{15}$

\section{Inotuzumab ozogamicin clinical experience in B cell lineage ALL}

The clinical experience using inotuzumab ozogamicin in patients with B cell non-Hodgkin's lymphoma has been previously extensively reviewed..$^{32,33}$ The encouraging results observed with inotuzumab ozogamicin in this pathology have led to investigations into inotuzumab ozogamicin therapy in other B cell lineage diseases, including ALL. Most of the published experiences with inotuzumab ozogamicin in ALL come from studies initiated at the MD Anderson Cancer Center in Houston (Texas, USA). ${ }^{34}$

\section{Inotuzumab ozogamicin as single agent in the treatment of relapsed/refractory ALL}

In a first report, inotuzumab ozogamicin appears as one of the single most active single agent tested in refractory or relapsed B cell lineage ALL. ${ }^{15}$ Forty-nine patients with refractory or relapsed B cell lineage ALL, aged from 6 to 80 years (median, 36 years), received inotuzumab ozogamicin at $1.8 \mathrm{mg} / \mathrm{m}^{2}$ intravenously over 1 hour every 3 weeks, the maximum tolerated dose determined in B cell lymphoma. All patients expressed high levels of CD22 positivity in $\geq 50 \%$ leukemic cells, and for 20 patients $\geq 90 \%$ in leukemic cells. Overall, a response (complete marrow response, complete marrow response without platelet recovery, and complete marrow response with incomplete recovery of peripheral blood cell counts) was observed in $57 \%$ of cases $(66 \%$ in second line therapy, $48 \%$ in third line therapy, and $40 \%$ in further lines of therapy). Patients with Philadelphia chromosome positive ALL and translocation (4;11) were less likely to respond than patients with other karyotypes. Three out of the seven patients with Philadelphia chromosome positive ALL achieved morphologic and cytogenetic complete responses. The lowest BCR-ABL transcript levels were $0.09 \%, 0.01 \%$, and $0.07 \%$, respectively. The median number of treatment courses was two (range, 1-5 courses) and the median time between courses was 3 weeks (range, 3-6 weeks). Eighty-two percent of patients received two courses or more and $47 \%$ received three or more. Nine (18\%) patients received rituximab with their third treatment course, of whom only one showed 
a response (after the fourth course). The median overall survival was 5.1 months. Median survival for responders was 7.9 months. Eighty-nine percent of responders with initial chromosomal abnormalities achieved a complete cytogenetic response. A negative minimal residual disease (MRD) status was observed in $63 \%$ of morphologic responders. Fever and mild hypotension occurred in most patients on days 1-2 and resolved with support. Fever was severe (grade 3-4) in 31\% of these cases and hypotension was observed in $2 \%$ of cases. Liver function abnormalities were noted in $25 \%$ of cases, but were severe in only half of those cases with periportal fibrosis observed in liver biopsies in two patients.

After the initial report using an every 3- to 4-week schedule, the schedule was modified to give inotuzumab ozogamicin weekly at $0.8 \mathrm{mg} / \mathrm{m}^{2}$ on day 1 followed by $0.5 \mathrm{mg} / \mathrm{m}^{2}$ on days 8 and 15 every 3 to 4 weeks, based on higher in vitro efficacy with more frequent exposure. ${ }^{34}$ Forty-one patients were studied. Eight patients (20\%) achieved complete response, 13 patients $(32 \%)$ achieved bone marrow complete response with incomplete recovery of platelets, and 3 patients (7\%) achieved bone marrow complete response with incomplete recovery. There were no differences regarding MRD negative achievement according to whether patients received weekly or single dose inotuzumab ozogamicin. Higher inotuzumab ozogamicin peak levels were observed with the single dose schedule, but peak levels did not correlate with response rates. The weekly dosing schedule was associated with less hepatic toxicity as compared with the single dose schedule given every 3 to 4 weeks. Fever and hypotension within 48 hours of drug administration were also observed less frequently on the weekly dosing schedule. The median survival was 5 months with the single dose schedule and 7.3 months with the weekly schedule (Table 1).

The encouraging clinical activity of single agent inotuzumab ozogamicin in a relapsed/refractory ALL population was confirmed in another recent study.$^{35}$ Preliminary efficacy results appeared to be dynamically related to inotuzumab ozogamicin exposure, cytogenetics, and circulating blasts. In this dose escalation trial, inotuzumab ozogamicin was administered on days 1,8 , and 15 over a 28 day cycle (up to six cycles). Total doses per cycle ranged from $1.2 \mathrm{mg} / \mathrm{m}^{2}$ to $2 \mathrm{mg} / \mathrm{m}^{2}$. Overall, 79\% of patients achieved a complete response $(46 \%$ of complete remission and $33 \%$ of bone marrow complete response with incomplete recovery of blood cell count). Ninety-five percent of them were shown to be MRD negative. Median time to hematologic response was 28 days (range, 20-78 days). The recommended Phase II dose was determined to be $1.8 \mathrm{mg} / \mathrm{m}^{2} /$ cycle based on safety and efficacy
Table I Characteristics and outcomes of relapsed/refractory acute lymphoblastic leukemia patients treated with inotuzumab ozogamicin used as a single agent

\begin{tabular}{|c|c|c|c|}
\hline Characteristics & $\begin{array}{l}\text { Single dose } \\
\text { schedule } \\
49 \text { patients }\end{array}$ & $\begin{array}{l}\text { Weekly } \\
\text { schedule } \\
41 \text { patients }\end{array}$ & $\begin{array}{l}\text { Overall } \\
90 \text { patients }\end{array}$ \\
\hline \multicolumn{4}{|l|}{ ECOG PS } \\
\hline $0-1$ & $44(90 \%)^{*}$ & $37(90 \%)$ & 81 (90\%) \\
\hline$\geq 2$ & $5(10 \%)$ & $4(10 \%)$ & $9(10 \%)$ \\
\hline \multicolumn{4}{|l|}{ Salvage status } \\
\hline First salvage & I3 (27\%) & $16(39 \%)$ & $29(32 \%)$ \\
\hline Second salvage & $24(49 \%)$ & $10(24 \%)$ & $34(38 \%)$ \\
\hline Further salvage & $12(24 \%)$ & $15(37 \%)$ & $27(30 \%)$ \\
\hline \multicolumn{4}{|l|}{ Karyotype } \\
\hline Diploid & $12(24 \%)$ & $9(22 \%)$ & $21(23 \%)$ \\
\hline $\mathrm{T}(9 ; 22)$ & $7(14 \%)$ & $8(20 \%)$ & $15(17 \%)$ \\
\hline $\mathrm{T}(4 ; \mathrm{II})$ & $5(10 \%)$ & $3(7 \%)$ & $8(9 \%)$ \\
\hline Other & $25(51 \%)$ & $21(51 \%)$ & $46(51 \%)$ \\
\hline \multicolumn{4}{|l|}{ CD22 positivity } \\
\hline$>90 \%$ & $28(57 \%)$ & 31 (76\%) & $59(66 \%)$ \\
\hline $70 \%-89 \%$ & $14(29 \%)$ & $8(20 \%)$ & $22(24 \%)$ \\
\hline $50 \%-69 \%$ & $7(\mid 4 \%)$ & $2(5 \%)$ & $9(10 \%)$ \\
\hline \multicolumn{4}{|l|}{ Response to therapy } \\
\hline CR & $9(18 \%)$ & $8(20 \%)$ & 17 (19\%) \\
\hline CRp & $14(29 \%)$ & $13(32 \%)$ & $27(30 \%)$ \\
\hline $\mathrm{CRi}$ & $5(10 \%)$ & $3(7 \%)$ & $8(9 \%)$ \\
\hline PR & 0 & 0 & 0 \\
\hline Resistant & $19(39 \%)$ & $15(37 \%)$ & $34(38 \%)$ \\
\hline Death within 4 weeks & $2(4 \%)$ & $2(5 \%)$ & $4(4 \%)$ \\
\hline
\end{tabular}

Notes: $*_{n}(\%)$. Adapted with permission from John Wiley \& Sons, Inc. Kantarjian $\mathrm{H}$, Thomas D, Jorgensen J, et al. Results of inotuzumab ozogamicin, a CD22 monoclonal antibody, in refractory and relapsed acute lymphocytic leukemia. Cancer. 2013;1 19: 2728-2736. () 2013 American Cancer Society. ${ }^{34}$

Abbreviations: $\mathrm{CR}$, complete response; $\mathrm{CRi}$, bone marrow complete response with incomplete recovery; CRp, bone marrow complete response with incomplete recovery of platelets; ECOG PS, Eastern Cooperative Oncology Group performance status; $\mathrm{PR}$, partial response.

results. The tolerable safety profile, primarily characterized by hematologic, gastrointestinal, and hepatic side effects, was consistent with that of prior reports. Fifty percent of patients underwent follow-up transplant after inotuzumab ozogamicin treatment. Among them, three patients died from sepsis. No events of veno-occlusive disease (VOD) were reported.

\section{Feasibility of subsequent allogeneic SCT}

After relapse, allogeneic SCT currently provides the most effective approach for inducing long term disease control. ${ }^{3,5,36}$ The overall survival rate was $16 \%-23 \%$ for those who received allogeneic SCT, compared with $0 \%-4 \%$ for those who received chemotherapy alone. ${ }^{3,36}$ Because many patients have had previous exposure to chemotherapy drugs used for salvage chemotherapy and have experienced toxicity resulting from dose intense therapy, antibody therapy with a nonoverlapping toxicity and a different mechanism of action from drugs used in front line therapy can represent an ideal 
therapy in the relapsed situation. The high response rate observed with inotuzumab ozogamicin used as a single agent enabled subsequent allogeneic SCT in a large number of patients $(45 \%),{ }^{15,37}$ of which a high percentage were MRD negative at the time of the transplant. The median time from the end of inotuzumab ozogamicin therapy to allogeneic SCT was 5 weeks (range, 2-14 weeks). ${ }^{37}$ Regarding toxicity of the transplant procedure, transient liver enzyme elevations was noted in most of the patients. Similar findings were noted after therapy with gemtuzumab ozogamicin, an anti-CD33 calicheamicin conjugate, which was previously approved for treatment of relapsed/refractory acute myeloid leukemia. ${ }^{16}$ However, liver function abnormalities have also been observed in the nontransplant setting. ${ }^{37}$ Antibody drug conjugates that employ calicheamicin have been associated with VOD. ${ }^{16}$ This toxicity is especially worrisome since the goal of treatment after CR achievement is to proceed to allogeneic SCT. After inotuzumab ozogamicin administration, some patients developed VOD: one case among the 14 patients who underwent allogeneic SCT after weekly inotuzumab ozogamicin and five cases among the 22 transplanted patients after single dose inotuzumab ozogamicin. ${ }^{34}$ The incidence of VOD was higher than that typically noted for allogeneic SCT in ALL. This was associated with the number of lines of prior therapy (more toxicity in patients with three to five lines prior SCT), and more intensive transplant conditioning regimens (busulfan-clofarabine-thiotepa or fludarabine-melphalan-thiotepa). Transplant preparative regimens that minimize liver toxicity should therefore be preferred after inotuzumab ozogamicin therapy. The interval between inotuzumab ozogamicin administration and the start of SCT conditioning regimen did not appear to influence the risk of VOD developing. ${ }^{37}$ There does not appear to be a correlation between inotuzumab ozogamicin administration and the risk for graft versus host disease developing.

\section{Inotuzumab ozogamicin compared to chemotherapy in patients with relapsed/refractory ALL}

The outcome of the 90 patients with relapsed or refractory ALL treated with inotuzumab ozogamicin used as a single agent ${ }^{34}$ was compared to that of historical controls consisting in 292 ALL patients with the same leukemia status treated with chemotherapy alone between 1990 and 2008 in the same institution. ${ }^{38}$ Outcomes after single agent inotuzumab ozogamicin were better than those after treatment with chemotherapy alone. In first salvage, complete response was significantly better with inotuzumab ozogamicin ( $66 \%$ versus $40 \% ; P=0.007)$. It was similar for patients in second salvage (44\% versus $16 \%$; $P<0.001$ ), third salvage ( $46 \%$ versus $19 \% ; P=0.03$ ), and fourth salvage $(27 \%$ versus $9 \% ; P=0.01) .{ }^{38}$ Overall survival was also better after inotuzumab ozogamicin.

\section{Experience with inotuzumab ozogamicin in pediatric patients with relapsed ALL} Inotuzumab ozogamicin was evaluated in pediatric patients with multiply relapsed ALL. ${ }^{39}$ Five children with relapsed/ refractory $\mathrm{CD} 22$ positive $B$ cell lineage ALL were treated with inotuzumab ozogamicin initially administered at $1.3 \mathrm{mg} / \mathrm{m}^{2}$ every 3 weeks. Then the dose was increased to $1.8 \mathrm{mg} / \mathrm{m}^{2}$ every 3 weeks. Subsequently, a weekly schedule was given as $0.8 \mathrm{mg} / \mathrm{m}^{2}$ on day 1 followed by $0.5 \mathrm{mg} / \mathrm{m}^{2}$ on days 8 and 15. One patient achieved complete response and two patients obtained bone marrow complete response with incomplete recovery of platelets. The last two patients had no response to the drug. Inotuzumab ozogamicin was relatively well tolerated. Three pediatric patients proceeded to transplant following inotuzumab ozogamicin therapy. One developed VOD following unrelated donor transplantation. This episode of VOD resolved after treatment with defibrotide.

\section{Combination of inotuzumab ozogamicin with chemotherapy in patients with ALL}

Eleven patients, aged 60 years or older with newly diagnosed B cell lineage ALL, were treated with a combination of low intensity hyper-CVAD (Cyclophosphamide, Vincristine, Adriamycin, Dexamethasone) chemotherapy (cyclophosphamide and dexamethasone at 50\% dose reduction, no anthracycline, methotrexate at $75 \%$ dose reduction, and cytarabine at $0.5 \mathrm{~g} / \mathrm{m}^{2}$ for four doses), rituximab, intrathecal therapy, and inotuzumab ozogamicin (on day 3 of each cycle) for four cycles. ${ }^{40}$ The first six patients received inotuzumab ozogamicin at $1.3 \mathrm{mg} / \mathrm{m}^{2}$ for cycle one followed by $1.3 \mathrm{mg} / \mathrm{m}^{2}$ for subsequent cycles, while the others received $1.8 \mathrm{mg} / \mathrm{m}^{2}$ for cycle one followed by $1.3 \mathrm{mg} / \mathrm{m}^{2}$ for subsequent cycles. ${ }^{40}$ Ten patients (91\%) achieved morphological complete remission and flow cytometric MRD negative status. Six month disease free and overall survival were both $90 \%$. No dose limiting toxicity was observed.

\section{Conclusion}

First trials using inotuzumab ozogamicine in the treatment of B cell lineage ALL have shown impressive response rates in the relapsed/refractory setting and appear promising in improving outcomes. A weekly dose schedule appeared to be equally effective (59\% versus $57 \%$ of response rate) and less toxic than a single dose schedule every 3 weeks. ${ }^{34}$ The ability 
to achieve MRD negative responses has also been particularly impressive. Although high response rates were observed, studies with single agent inotuzumab ozogamicin showed that responses were not durable and that median survival was modest. However, results compared favorably to those observed in historic controls regarding relapsed/refractory ALL patients treated by chemotherapy. ${ }^{41,42}$ Thus, MoAb potentially offers highly active modalities to obtain better response than those observed after chemotherapy, but with less toxicity. Older patients with ALL have a poor outcome.$^{43-47}$ This is primarily due to the characteristics of ALL in this age population and to a poor tolerance of intensive chemotherapy which results in ineffective delivery of treatments. Combination of inotuzumab ozogamicin with low intensity chemotherapy has recently shown encouraging results in elderly patients with ALL. ${ }^{40}$ These first results appeared to be better than those obtained with chemotherapy alone and may become a new standard of care for the treatment of elderly patients with ALL. Future studies in the newly diagnosed setting and/or in combination with chemotherapy are awaited to evaluate whether the quality of response rates can also be improved in younger adults. Due to the encouraging response rate in adults, inotuzumab ozogamicin also appeared to be an attractive option for pediatric patients with relapsed ALL not responding to conventional chemotherapy. Although the maximum tolerated dose for pediatric patients was not explored, the drug appeared to be well tolerated using doses known to be safe in adults. ${ }^{39}$

Studies with inotuzumab ozogamicin in the treatment of ALL are currently ongoing. Results of two major studies are awaited: a larger Phase I trial to evaluate pharmacokinetics, levels of MRD, and response is ongoing (B1931010 trial), and a Phase III pivotal trial of inotuzumab ozogamicin versus investigator's choice of chemotherapy in adult patients with relapsed or refractory CD22 positive ALL which is still in progress (B1931022 trial).

Overall, the current review indicates that inotuzumab ozogamicin is effective to treat relapsed or refractory ALL. When optimum dose and scheduling is defined, inotuzumab could probably find a place in front line therapy. Its efficacy should also be compared with the promising recent results obtained with MoAb directed against CD20 and CD19 antigens.

\section{Disclosure}

The author reports no conflict of interest in this work.

\section{References}

1. Huguet F, Leguay T, Raffoux E, et al. Pediatric-inspired therapy in adults with Philadelphia chromosome-negative acute lymphoblastic leukemia: the GRAALL-2003 study. J Clin Oncol. 2009;27:911-918.
2. Rijneveld AW, van der Holt B, Daenen SM, et al; Dutch-Belgian HOVON Cooperative group. Intensified chemotherapy inspired by a pediatric regimen combined with allogeneic transplantation in adult patients with acute lymphoblastic leukemia up to the age of 40 . Leukemia. 2011;25:1697-1703.

3. Fielding AK, Richards SM, Chopra R, et al; Eastern Cooperative Oncology Group. Outcome of 609 adults after relapse of acute lymphoblastic leukemia (ALL); an MRC UKALL 12/ECOG 2993 study. Blood. 2007;109:944-950.

4. Oriol A, Vives S, Hernandez-Rivas JM, et al; Programa Espanol de Tratamienti en Hematologia Group. Outcome after relapse of acute lymphoblastic leukemia in adult patients included in four consecutive risk-adapted trials by the PETHEMA Study Group. Haematologica. 2010;95:589-596.

5. Tavernier E, Boiron JM, Huguet F, et al; GET-LALA Group; Swiss Group for Clinical Cancer Research SAKK; Australasian Leukemia and Lymphoma Group. Outcome of treatment after first relapse in adults with acute lymphoblastic leukemia initially treated by the LALA-94 trial. Leukemia. 2007;21:1907-1914.

6. Hoelzer D. Novel antibody-based therapies for acute lymphoblastic leukemia. Hematology Am Soc Hematol Educ Program. 2011;2011: 243-249.

7. Dubowchik G, Walker M. Receptor-mediated and enzyme-dependent targeting of cytotoxic anti-cancer drugs. Pharmacol Ther. 1999;83: 67-123.

8. Leonard JP, Goldenberg DM. Preclinical and clinical evaluation of epratuzumab (anti-CD22 IgG) in B-cell malignancies. Oncogene. 2007;26:3704-3713.

9. Tedder TF, Poe JC, Haas KM. CD22: a multifunctional receptor that regulates B lymphocyte survival and signal transduction. Adv Immunol. 2005;88:1-50.

10. Raponi S, Stefania de Propris M, Intoppa S, et al. Flow cytometry study of potential target antigens (CD19, CD20, CD22, CD33) for antibodybased immunotherapy in acute lymphoblastic leukemia: analysis of 552 cases. Leuk Lymphoma. 2011;52:1098-1107.

11. Gudowius S, Recker K, Laws HJ, et al. Identification of candidate target antigens for antibody-based immunotherapy in childhood B-cell precursor ALL. Klin Pediatr. 2006;218:327-333.

12. Shan D, Press OW. Constitutive endocytosis and degradation of CD22 by human B cells. J Immunol. 1995;154:4466-4475.

13. Sato S, Tuscano JM, Inaoki M, Tedder TF. CD22 negatively and positively regulates signal transduction through the B lymphocyte antigen receptor. Semin Immunol. 1998;10:287-297.

14. von Stackelberg A. Monoclonal antibodies in pediatric acute lymphoblastic leukemia. In: Saha V, Kearns P, editors. New Agents for the Treatment of Acute Lymphoblastic Leukemia. New York: Springer; 2011:221-273.

15. Kantarjian $\mathrm{H}$, Thomas D, Jorgensen J, et al. Inotuzumab ozogamicin, an anti-CD22-calicheamicin conjugate, for refractory and relapsed acute lymphocytic leukaemia: a phase 2 study. Lancet. 2012;13:403-411.

16. Thorson J, Sievers E, Ahlert J, et al. Understanding and exploiting nature's chemical arsenal: the past, present and future of calicheamicin research. Curr Pharm Des. 2000;6:1841-1879.

17. Zein N, Sinha AM, McGahren WJ, Ellestag GA. Calicheamicin gamma 1I: an antitumor antibiotic that cleaves double-stranded DNA site specifically. Science. 1988;240:1198-1201.

18. Walker S, Landovitz R, Ding WD, Ellestad GA, Kahne D. Cleavage behavior of calicheamicin gamma 1 and calicheamicin T. Proc Natl Acad Sci U S A. 1992;89:4608-4612.

19. DiJoseph JF, Armellino DC, Bogaert ER, et al. Antibody-targeted chemotherapy with CMC-544: a CD22-targeted immunoconjugate of calicheamicin for the treatment of B lymphoid malignancies. Blood. 2004;103:1807-1814.

20. DiJoseph JF, Dougher MM, Kalyandrug LB, et al. Antitumor efficacy of a combination of CMC-544 (inotuzumab ozogamicin), a CD22-targeted cytotoxic immunoconjugate of calicheamicin, and rituximab against non-Hodgkin's B-cell lymphoma. Clin Cancer Res. 2006;12:242-249. 
21. Damle NK. Tumor-targeted chemotherapy using immunoconjugates of calicheamicin. Expert Opin Biol Ther. 2004;4:1445-1452.

22. Damle NK, Frost P. Antibody-targeted chemotherapy with immunoconjugates of calicheamicin. Curr Opin Pharmacol. 2003;3:386-390.

23. DiJoseph JF, Dougher MM, Armellino DC, Evans DY, Damle NK. Therapeutic potential of CD22-specific antibody-targeted chemotherapy using inotuzumab ozogamicin (CMC-544) for the treatment of acute lymphoblastic leukemia. Leukemia. 2007;21:2240-2245.

24. Zwaan CM, Reinhardt D, Jurgens H, et al. Gemtuzumab ozogamicin in pediatric CD33-positive acute lymphoblastic leukemia: first clinical experiences and relation with cellular sensitivity to single agent calicheamicin. Leukemia. 2003;17:468-470.

25. DiJoseph JF, Dougher MM, Evans DY, Zhou BB, Damle NK. Preclinical anti-tumor activity of antibody-targeted chemotherapy with CMC-544 (inotuzumab ozogamicin), a CD22-specific immunoconjugate of calicheamicin, compared with non-targeted chemotherapy CVP or CHOP. Cancer Chemother Pharmacol. 2011;67:741-749.

26. de Vries JF, Zwaan CM, De Bie M, et al. The novel calicheamicinconjugated CD22 antibody inotuzumab ozogamicin (CMC-544) effectively kills primary pediatric acute lymphoblastic leukemia cells. Leukemia. 2012;26:255-264.

27. DiJoseph JF, Goad ME, Dougher MM, et al. Potent and specific antitumor efficacy of CMC-544, a CD22-targeted immunoconjugate of calicheamicin, against systemically disseminated B-cell lymphoma. Clin Cancer Res. 2004;10:8620-8629.

28. Takeshita A, Shinjo K, Yamakage N, et al. CMC-544 (inotuzumab ozogamicin) shows less effect on multidrug resistant cells: analyses in cell lines and cells from patients with B-cell chronic lymphocytic leukaemia and lymphoma. Br J Haematol. 2009;146:34-43.

29. Takeshita A, Yamakage N, Shinjo K, et al. CMC-544 (inotuzumab ozogamicin), an anti-CD22 immuno-conjugate of calicheamicin, alters the levels of target molecules of malignant B-cells. Leukemia. 2009;23: 1329-1336.

30. DiJoseph JF, Dougher MM, Kalyandrug L, Damle NK. Anti-tumor activity of CMC-544 in combination with rituximab. Proc Am Assoc Cancer Res. 2005;46:abstr 700.

31. Macor P, Tripodo C, Zorzet S, et al. In vivo targeting of human neutralizing antibodies against CD55 and CD59 to lymphoma cells increases the antitumor activity of rituximab. Cancer Res. 2007;67:10556-10563.

32. Wong BY, Dang NH. Inotuzumab ozogamicin as novel therapy in lymphomas. Expert Opin Biol Ther. 2010;10:1251-1258.

33. Ricart AD. Antibody-drug conjugates of calicheamicin derivative: gemtuzumab ozogamicin and inotuzumab ozogamicin. Clin Cancer Res. 2011;17:6417-6427.

34. Kantarjian H, Thomas D, Jorgensen J, et al. Results of inotuzumab ozogamicin, a CD22 monoclonal antibody, in refractory and relapsed acute lymphocytic leukemia. Cancer. 2013;119:2728-2736.

35. DeAngelo DJ, Stock W, Petersdorf S, et al. Weekly inotuzumab ozogamicin in adult patients with relapsed or refractory CD22-positive acute lymphoblastic leukemia. Blood. 2012;suppl 120:abstr 2612.
36. Kozlowski P, Aström M, Ahlberg L, et al. High curability via intensive reinduction chemotherapy and stem cell transplantation in young adults with relapsed acute lymphoblastic leukemia in Sweden 2003-2007. Haematologica. 2012;97:1414-1421.

37. Kebriaei P, Wilhelm K, Ravandi F, et al. Feasibility of allografting in patients with advanced acute lymphoblastic leukemia after salvage therapy with inotuzumab ozogamicin. Clin Lymph Myel Leuk. 2013;13: 296-301.

38. Faderl S, Jain N, O’Brien SM, et al. Inotuzumab ozogamicin (CMC-544) compared to chemotherapy in patients (pts) with relapsed/refractory (R/R) acute lymphoblastic leukemia (ALL): a retrospective comparison. J Clin Oncol. 2013;suppl 31:abstr 7095. ASCO 2013.

39. Rytting M, Triche L, Thomas D, O’Brien S, Kantarjian H. Initial experience with CMC-544 (inotuzumab ozogamicin) in pediatric patients with relapsed B-cell acute lymphoblastic leukemia. Pediatr Blood Cancer. 2014;61:369-372.

40. Jain N, O'Brien SM, Thomas DA, et al. Phase I/II study of the combination of inotuzumab ozogamicin (CMC-544) with low-intensity chemotherapy in patients (pts) with acute lymphoblastic leukemia (ALL). J Clin Oncol. 2013;suppl 31:abstr 7092. ASCO 2013.

41. O'Brien S, Thomas D, Ravandi F, et al. Outcome of adults with acute lymphocytic leukemia after second salvage therapy. Cancer. 2008;113: 3186-3191.

42. Thomas DA, Kantarjian H, Smith TL, et al. Primary refractory and relapsed adult acute lymphoblastic leukemia: characteristics, treatment results, and prognosis with salvage therapy. Cancer. 1999;86: 1216-1230.

43. Delannoy A, Sebban C, Cony-Makhoul P, et al. Age-adapted induction treatment of acute lymphoblastic leukemia in the elderly and assessment of maintenance with interferon combined with chemotherapy. A multicentric prospective study in forty patients. French Group for Treatment of Adult Acute Lymphoblastic Leukemia. Leukemia. 1997;11:1429-1434.

44. Bassan R, Di Bona E, Lerede T, et al. Age-adapted moderate-dose induction and flexible outpatient post-remission therapy for elderly patients with acute lymphoblastic leukemia. Leuk Lymphoma. 1996;22: 295-301.

45. Kantarjian HM, O’Brien S, Smith T, et al. Acute lymphocytic leukaemia in the elderly: characteristics and outcome with the vincristineadriamycin-dexamethasone (VAD) regimen. Br J Haematol. 1994;88: 94-100.

46. Sancho JM, Ribera JM, Xicoy B, et al; PETHEMA Group. Results of the PETHEMA ALL-96 trial in elderly patients with Philadelphia chromosome-negative acute lymphoblastic leukemia. Eur J Haematol. 2007;78:102-110.

47. Thomas X, Olteanu N, Charrin C, Lheritier V, Magaud JP, Fiere D. Acute lymphoblastic leukemia in the elderly: The Edouard Herriot Hospital experience. Am J Hematol. 2001;67:73-83.
Blood and Lymphatic Cancer: Targets and Therapy

\section{Publish your work in this journal}

Blood and Lymphatic Cancer: Targets and Therapy is an international, peer-reviewed, open access journal focusing on blood and lymphatic cancer research, identification of therapeutic targets and the optimal use of preventative and integrated treatment interventions to achieve improved outcomes, enhanced survival and quality of life for the
Dovepress

cancer patient. The manuscript management system is completely online and includes a very quick and fair peer-review system. Visit http://www.dovepress.com/testimonials.php to read real quotes from published authors. 